\title{
A deceptive case of emphysematous cholecystitis complicated with retroperitoneal gangrene and emphysematous pancreatitis: clinical and computed tomography features
}

\author{
Małgorzata Deręgowska-Cylke ${ }^{1 A, B, D, E, F}$, Piotr Palczewski ${ }^{1 A, B, D, E, F}$, Marek Pacholczyk ${ }^{2 B, D}$, Maurycy Jonas ${ }^{2 B, D}$, \\ Radosław Cylke $2 B, D$, Maciej Kosieradzki2B,D ${ }^{2}$, Marek Gołębiowski ${ }^{1 A, D, E}$ \\ ${ }^{1} 1^{\text {st }}$ Department of Clinical Radiology, Medical University of Warsaw, Poland \\ 2Department of General and Transplantation Surgery, Medical University of Warsaw, Poland
}

\section{Abstract}

Purpose: Emphysematous cholecystitis (EC) is an uncommon, severe variant of acute cholecystitis caused by gasforming bacteria - most often Clostridium perfringens and Escherichia coli. We present a deceptive case of EC associated with retroperitoneal gas gangrene and emphysematous pancreatitis.

Case report: An 86-year-old, overweight woman was admitted to the emergency department with non-specific abdominal symptoms. Admission laboratory tests showed elevated diastase levels indicating acute pancreatitis. Computed tomography (CT) demonstrated a substantial amount of gas in the retroperitoneum and peritoneal cavity, which raised a suspicion of duodenal perforation. Primary diagnosis was not confirmed during emergency laparotomy, which revealed a gangrenous gallbladder adjacent to the duodenum and surrounded by purulent fluid. The final diagnosis established after laparotomy and rereading of CT scans was that of emphysematous cholecystitis associated with gangrenous pancreatitis and retroperitoneal gangrene. After surgery, the patient was transferred to the intensive care unit in septic shock. Shortly after, the second laparotomy was undertaken on suspicion of internal bleeding. During surgery, the patient experienced cardiac arrest and died despite immediate resuscitation.

Conclusions: Emphysematous cholecystitis may be associated with a spread of infection both to the peritoneal cavity and retroperitoneum and result in a substantial amount of gas in those anatomic compartments. The knowledge of this rare complication may be helpful in establishing a correct diagnosis.

Key words: pancreatitis, pneumoperitoneum, multidetector computed tomography, emphysematous cholecystitis, fasciitis, necrotising.

\section{Introduction}

Emphysematous cholecystitis (EC) is an uncommon, severe variant of acute cholecystitis caused by gas-forming bacteria, most often Clostridium perfringens and Escherichia coli [1-5]. Characterised by a propensity for an early gangrene and gallbladder perforation with a resultant high mortality, EC is a recognised surgical emergency [3].
We present a case of fatal EC complicated with retroperitoneal gangrene and emphysematous pancreatitis, which posed a diagnostic challenge due to deceptive laboratory test results and imaging findings. Emphysematous cholecystitis as well as pancreatitis are commonly described in the literature; however, the combination of those two diseases in one patient is extremely rare.

Correspondence address:

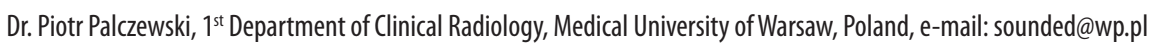

Authors' contribution:

A Study design · B Data collection · C Statistical analysis · D Data interpretation - E Manuscript preparation · F Literature search · G Funds collection 


\section{Case report}

An 86-year-old, overweight $(\mathrm{BMI}=29)$ woman presented to the emergency department with a severe abdominal pain radiating to the back, nausea, and vomiting for one day. Her medical history included an appendectomy at the age of 13 years and the implantation of heart pacemaker the previous year. The patient's vital signs were as follows: heart rate: $88 / \mathrm{min}$, blood pressure: $110 / 50 \mathrm{mmHg}$, saturation: $98 \%$ in room air, and body temperature: $36.6^{\circ} \mathrm{C}$. The physical examination revealed signs of dehydration and tenderness localised in the middle upper abdomen, without signs of peritoneal irritation and with clearly audible peristalsis. The results of the laboratory tests showed elevation of pancreatic enzymes: serum amylase (1218 U/l), urinary amylase (8100 U/l), and serum lipase (5850 U/l). Glucose level was elevated (175 mg/dl); however, the patient had not been diagnosed with diabetes previously and did not take any diabetes medications. The remaining laboratory tests, including serum C-reactive protein level $(2.1 \mathrm{mg} / \mathrm{l})$ and serum white blood cell count $\left(9.57 \times 10^{9} / \mathrm{l}\right)$, were within normal range. Admission abdominal ultrasound showed marginal dilatation of the common bile duct $(9 \mathrm{~mm})$; however, intrahepatic bile ducts were not dilated. The gallbladder was described as thin-walled, with no intraluminal gallstones. The pancreas was impossible to evaluate due to excessive bowel gas overlying the organ. No intraperitoneal free fluid or free gas was detected during the examination. The patient was admitted to the surgery department with a suspicion of acute pancreatitis and put on a strict diet, intravenous hydration, and analgesic treatment. Despite the treatment, the patient complained about persistent severe abdominal pain and developed hypotension and oliguria. At this stage, the laboratory tests showed slightly elevated serum C-reactive protein level $(48 \mathrm{mg} / \mathrm{l})$, decreased serum white blood cell count $\left(2.27 \times 10^{9} / 1\right)$ with high percentage of neutrophils $(77.7 \%)$, and increased serum aspartate aminotransferase (59 U/l) and alanine aminotransferase (53 U/l). Levels of amylase and lipase were comparable to the initial results. Due to deteriorating general condition, the patient was scheduled to undergo computed tomography (CT) of the abdomen, which was performed 16 hours after admission ultrasound. Based on the presence of free gas in the peritoneal cavity and retroperitoneally, mostly around the duodenum, pancreas, and visceral surface of the liver (Figure 1A), a radiologist on duty suggested a perforated duodenal diverticulum (Figure 1C), and a decision was made to perform an emergency surgery. During laparotomy, perforation of the duodenum was ruled out; however, adjacent to the duodenum there was a significantly enlarged gangrenous gallbladder, surrounded by free fluid of purulent character. Therefore, a cholecystectomy was performed followed by peritoneal cavity lavage and drainage. Retrospective analysis of CT images revealed gas in the gallbladder wall and within pancreatic parenchyma
(Figure 1D-E). Pneumobilia in the left hepatic lobe and penetration of gas into abdominal wall (external oblique, transverse abdominal, and quadratus lumborum muscles) were considered atypical for acute duodenal perforation. Therefore, the final diagnosis established after laparotomy and rereading of CT scans was that of emphysematous cholecystitis associated with gangrenous pancreatitis and retroperitoneal gangrene. Postoperatively, the patient was transferred to the intensive care unit in critical condition, with signs of septic shock and multiorgan failure. Besides increased levels of procalcitonin $(38.96 \mathrm{ng} / \mathrm{ml})$, C-reactive protein $(118.2 \mathrm{mg} / \mathrm{l}), \mathrm{D}$-dimer $(2000.0 \mathrm{ng} / \mathrm{ml})$, lactate dehydrogenase (1290 U/l), creatine phosphokinase (3787 U/l), myoglobin (> $12000 \mathrm{ng} / \mathrm{ml})$, and troponin $(0.018 \mathrm{ng} / \mathrm{ml})$, the laboratory results showed decreased red blood cell count $\left(3.4410^{12} / \mathrm{l}\right)$ and haemoglobin level $(107.0 \mathrm{~g} / \mathrm{l})$, which led to relaparotomy to exclude intraperitoneal bleeding. During the surgery cardiac arrest occurred and despite immediate resuscitation the patient died.

\section{Discussion}

First clinical descriptions of emphysematous cholecystitis date back to the turn of the $19^{\text {th }}$ and $20^{\text {th }}$ centuries $[2,6]$. It was recognised early that this variant of acute cholecystitis is characterised by significant morbidity and mortality (up to $15-20 \%$ ) related to frequent gallbladder wall necrosis and perforation, the latter occurring twice as often as in uncomplicated acute cholecystitis [7]. EC is commonly associated with diabetes mellitus and has a greater incidence in men aged 50-70 years (often with peripheral atherosclerotic disease) than in women, who are generally more susceptible to acute cholecystitis without narrow age range [1]. Gallstones are found only in $40 \%$ of cases of EC, while in a classic acute cholecystitis they are present in about $90 \%$ of cases [1]. Early on, clinical symptoms consist of pain in the right upper abdomen, vomiting, and fever, and are indistinguishable from classic cholecystitis; however, when disease extends beyond the gallbladder, patients may rapidly deteriorate, developing septic shock and multiorgan failure $[4,5,8]$. Because of this initially insidious course of EC, diagnostic imaging plays an important role in its early detection.

The hallmark of EC is the production of gas by anaerobic or facultatively anaerobic bacteria, such as Clostridium perfringens, Escherichia coli, Aerobacter aerogens, Klebsiella, and Salmonella spp. [1-5]. Gill et al. recognised three radiographic stages of EC based on the presence of gas in the gallbladder lumen (stage 1), the gallbladder wall (stage 2), and the pericholecystic tissues (stage 3 ) in the absence of an abnormal connection between biliary ducts and gastrointestinal tract [9]. Computed tomography has a higher accuracy in the detection of gas and in establishing its exact location than conventional radiography and ultrasonography [9]. Infrequently, the amount of gas produced by bacteria may lead to a massive pneumoperi- 

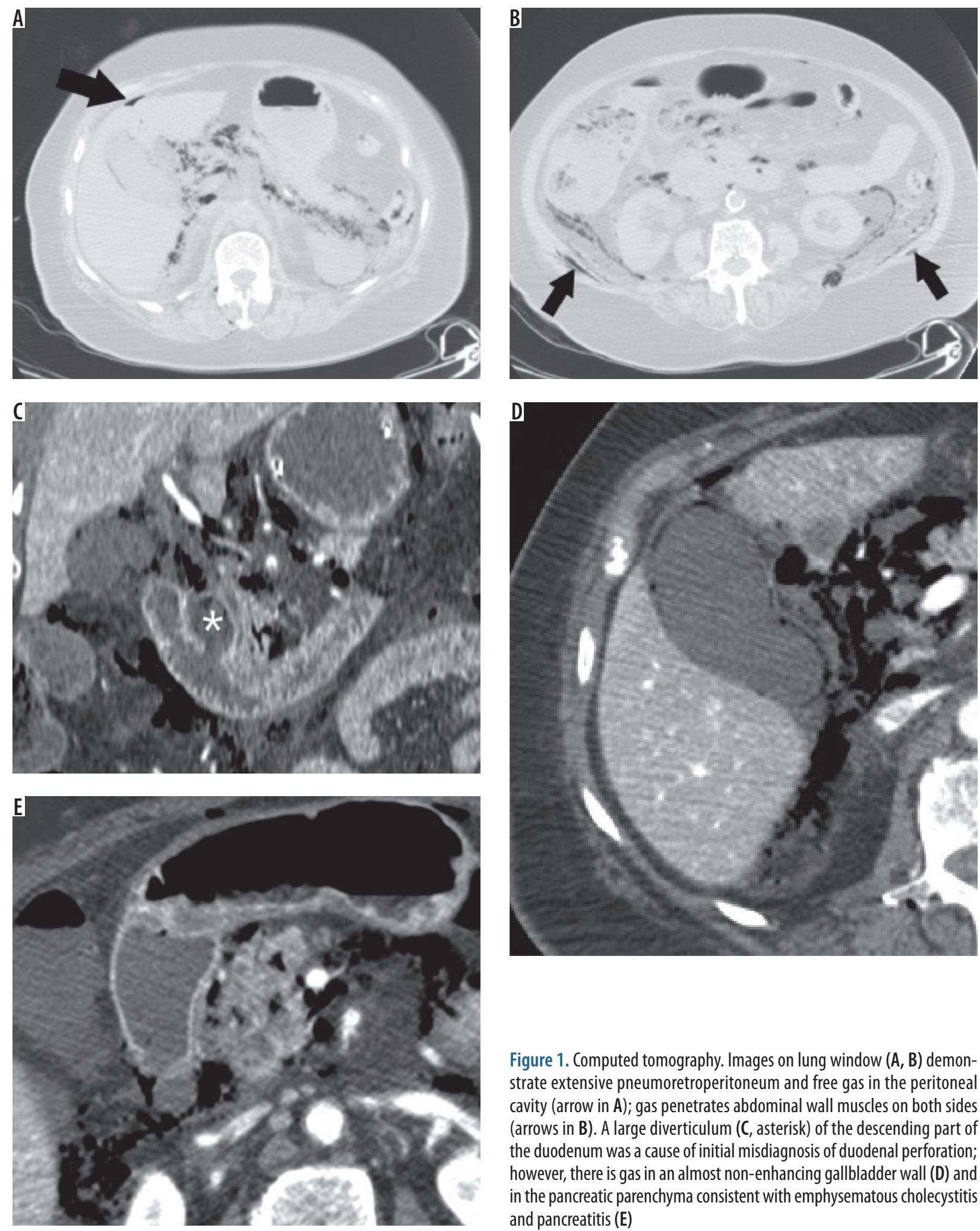

Figure 1. Computed tomography. Images on lung window (A, B) demonstrate extensive pneumoretroperitoneum and free gas in the peritoneal cavity (arrow in A); gas penetrates abdominal wall muscles on both sides (arrows in B). A large diverticulum (C, asterisk) of the descending part of the duodenum was a cause of initial misdiagnosis of duodenal perforation; however, there is gas in an almost non-enhancing gallbladder wall (D) and in the pancreatic parenchyma consistent with emphysematous cholecystitis and pancreatitis (E)

toneum or pneumoretroperitoneum, which may require differential diagnosis with perforation of the gastrointestinal tract, especially of the duodenum $[10,11]$. Notably, pneumoperitoneum is associated with a macroscopic gallbladder wall perforation in less than half of the cases $[11,12]$. Besides of the large amount of gas in the retroperitoneum and peritoneal cavity, the diagnostic difficulties

in the presented case were also related to misleading laboratory test results that initially showed only an increase in pancreatic enzymes, suggesting acute pancreatitis. A close inspection of CT images revealed gas in the pancreatic parenchyma consistent with concurrent emphysematous pancreatitis. To the best of our knowledge, the coexistence of EC and emphysematous pancreatitis has been reported 
previously only once in a patient with biliary stones [13] The authors suggested that reflux of enteric organisms into the pancreatic duct and biliary tree associated with stones might have been responsible for simultaneous emphysematous pancreatitis and cholecystitis [13]. In the absence of biliary stones in our patient, the spread of infection to the pancreas probably occurred through peritoneal and fascial planes. Infectious processes in the peritoneal cavity and retroperitoneum sometimes travel through those anatomic pathways to reach distant sites; organisms causing tissue necrosis may also transgress fascial planes [14]. The extension of gas from pericholecystic tissues to retroperitoneum via hepatoduodenal ligament has been reported previously in patients with EC [10,15]. Delgadeo-Plasencia et al. described a case of EC leading to pneumoperitoneum, pneumomediastinum (via retroperitoneal route), and subcutaneous emphysema [2]. Safioleas et al. reported four cases of soft tissue gas gangrene arising from EC [16]. Therefore, we assume that the clinical and imaging features observed in our patient were a result of gas gangrene spreading from the gallbladder to the retroperitoneum and further into the abdominal wall.

On ultrasonography, gas in the gallbladder appears as echogenic spots with reverberation artefact or as broader bands of acoustic shadowing [3,9]. Nemcek et al. described "effervescent" or "champagne" sonographic sign in EC, which is explained as multiple, tiny echogenic foci rising from the dependent portion of the gallbladder like bubbles in a glass of champagne [17]. Several gallbladder conditions, including multiple gallstones, porcelain gallbladder, and adenomyomatosis, can make sonographic diagnosis of EC challenging [4]. In our patient, however, the gallbladder was described as unremarkable on admission US, which can only be explained by misdiagnosis or a rapid progression of disease in the time interval between US and CT. Despite the wide availability of US, CT is being used more and more extensively for the diagnosis of acute abdominal problems, including biliary emergencies [18]. Several papers investigated CT findings associated with gallbladder wall necrosis that may warrant a more aggressive therapeutic approach in patients with acute cholecystitis [19-21]. The findings most specific for gangrenous cholecystitis include: marked gallbladder distention (greater than $4 \mathrm{~cm}$ ), decreased or lack of mural enhancement, irregular wall thickening, gas in the wall or lumen, and pericholecystic fluid [19-21].

Treatment options in EC depend on the general condition of a patient and range from cholecystectomy (open or laparoscopic) in stable patients to percutaneous cholecystostomy with broad-spectrum intravenous antibiotics in unstable patients not amenable to surgery $[12,22]$. Recent studies have shown that in high-risk patients, both with cholecystitis and necrotising pancreatitis, early treatment with minimally invasive procedures reduces mortality and makes it possible either to delay open surgery until the patient's general condition is improved or even to cure some patients without open surgery [23-25].

\section{Conclusions}

Emphysematous cholecystitis may be associated with a spread of infection both to the peritoneal cavity and retroperitoneum and may result in a substantial amount of gas in those anatomic compartments. Although this is an uncommon situation, knowledge of this complication may warrant a close inspection of the gallbladder on the diagnostic imaging and point to a correct diagnosis preoperatively. The anatomic proximity of the gallbladder to the pancreas may result in a simultaneous emphysematous infection in both organs.

\section{Conflict of interest}

The authors report no conflict of interest.

\section{References}

1. Safwan M, Penny SM. Emphysematous cholecystitis: a deadly twist to a common disease. J Diagn Med Sonography 2016; 32: 131-137.

2. Delgado-Plasencia L, Gonzalez-Garcia I, Rodriguez-Gonzalez D, et al. Pneumomediastinum as a complication of emphysematous cholecystitis: case report. BMC Gastroenterol 2010; 10: 99.

3. Sunnapwar A, Raut AA, Nagar AM, et al. Emphysematous cholecystitis: Imaging findings in nine patients. Indian J Radiol Imaging 2011; 21: 142-146.

4. Grayson DE, Abbott RM, Levy AD, et al. Emphysematous infections of the abdomen and pelvis: a pictorial review. Radiographics 2002; 22: 543-561.

5. Garcia-Sancho Tellez L, Rodriguez-Montes JA, Fernandez de Lis S, et al. Acute emphysematous cholecystitis. Report of twenty cases. Hepatogastroenterology 1999; 46: 2144-2148.
6. Stolz A. Uber gasbildung in der gollinweger. Arch Pathol Anat 1901; 165: 90-123.

7. Jeffrey RB, Laing FC, Wong W, et al. Gangrenous cholecystitis: diagnosis by ultrasound. Radiology 1983; 148: 219-221.

8. Moanna A, Bajaj R, del Rio C. Emphysematous cholecystitis due to Salmonella derby. Lancet Infect Dis 2006; 6: 118-120.

9. Gill KS, Chapman AH, Weston MJ. The changing face of emphysematous cholecystitis. Br J Radiol 1997; 70: 986-991.

10. Yagi Y, Sasaki S, Terada I, et al. Massive pneumoretroperitoneum arising from emphysematous cholecystitis: a case report and the literature review. BMC Gastroenterol 2015; 15: 114.

11. Miyahara H, Shida D, Matsunaga H, et al. Emphysematous cholecystitis with massive gas in the abdominal cavity. World J Gastroenterol 2013; 19: 604-606. 
12. Zeebregts CJ, Wijffels RT, de Jong KP, et al. Percutaneous drainage of emphysematous cholecystitis associated with pneumoperitoneum. Hepatogastroenterology 1999; 46: 771-774.

13. Choi HS, Lee YS, Park SB, et al. Simultaneous emphysematous cholecystitis and emphysematous pancreatitis: a case report. Clin Imaging 2010; 34: 239-241.

14. Chingkoe CM, Jahed A, Loreto MP, et al. Retroperitoneal Fasciitis: Spectrum of CT Findings in the Abdomen and Pelvis. Radiographics 2015; 35: 1095-1107.

15. Catalano O. Nunziata A, Siani A. Emphysematous cholecystitis as a cause of retroperitoneal air. Emergency Radiology 2001; 8: 297-298.

16. Safioleas M, Stamatakos M, Kanakis M, et al. Soft tissue gas gangrene: a severe complication of emphysematous cholecystitis. Tohoku J Exp Med 2007; 213: 323-328.

17. Nemcek AA, Jr., Gore RM, Vogelzang RL, et al. The effervescent gallbladder: a sonographic sign of emphysematous cholecystitis. AJR Am J Roentgenol 1988; 150: 575-577.

18. Patel NB, Oto A, Thomas S. Multidetector CT of emergent biliary pathologic conditions. Radiographics 2013; 33: 1867-1888.

19. Bennett GL, Rusinek H, Lisi V, et al. CT findings in acute gangrenous cholecystitis. AJR Am J Roentgenol 2002; 178: 275-281.
20. Chang WC, Sun Y, Wu EH, et al. CT Findings for detecting the presence of gangrenous ischemia in cholecystitis. AJR Am J Roentgenol 2016; 207: 302-309.

21. Singh AK, Sagar P. Gangrenous cholecystitis: prediction with CT imaging. Abdom Imaging 2005; 30: 218-221.

22. Pal K. Laparoscopy in the management of emphysematous cholecystitis and secondary appendicitis in an 11-year-old child with insulin-dependent diabetes mellitus. Afr J Paediatr Surg 2011; 8: 211-214.

23. Šileikis A, Pečiulytė E, Misenkienè A, et al. Is minimally invasive surgical treatment justified for severe acute necrotizing pancreatitis patients with dysfunction of two or more organ systems? Wideochir Inne Tech Maloinwazyjne 2017; 12: 225-230.

24. Jagielski M, Smoczyński M, Adrych K. Endoscopic treatment of multilocular walled-off pancreatic necrosis with the multiple transluminal gateway technique. Wideochir Inne Tech Maloinwazyjne 2017; 12: 199-205.

25. Vázquez Tarragón A, Azagra Soria JS, Lens V, et al. Two staged minimally invasive treatment for acute cholecystitis in high risk patients. Hepatogastroenterology 2013; 60: 466-469. 